Varieties of decisional incapacity: theory and practice ${ }^{\dagger}$

\author{
Scott Y. H. Kim
}

\section{Summary}

Evaluation of decision-making capacity (DMC) for treatment is challenging. Owen et al, in this issue of the Journal, compare the abilities (understanding, appreciation and reasoning) relevant to DMC in medical and psychiatric patients. Here I discuss three key issues their article raises and that are relevant to the direction of future research.

Declaration of interest

None.
Scott Kim is faculty and senior investigator in the Department of Bioethics, National Institutes of Health, Bethesda, Maryland.

Patients' authority to make their own medical decisions is ordinarily presumed, but when there are concerns about potential decisional impairment, the modern practice is to assess whether they retain sufficient amount and type of abilities relevant to decision-making. This function-based framework - in contrast to an appeal to a diagnosis or a label ('unsound mind') - for determining patients' decision-making capacity (DMC) reflects the high priority we now place on patient self-determination. It is agreed (see the Mental Capacity Act 2005 and Grisso \& Appelbaum $^{1}$ ) that the functional abilities necessary for DMC include the abilities to understand (i.e. intellectually absorb) the relevant information and to communicate a choice. There is less agreement on what essential abilities lie between the taking in of information and the expression of choice. Depending on the jurisdiction, these abilities may include appreciation (ability to form adequate beliefs regarding their situation) and reasoning (using or manipulating the information in some minimally adequate way), ${ }^{1}$ or, as stated in the Mental Capacity Act 2005, 'using or weighing' information to reach a decision.

The law states these broad criteria with relatively sparse definitions and it expects each clinician evaluator to apply them much as a judge would, in effect approximating what a court's judgment would be. ${ }^{1}$ However, unlike a judge in a controlled court room, the busy clinician faces significant time constraints and higher frequency of cases, with little or no legal training. Treatment DMC evaluation is also different from other types of forensic evaluations because they are not usually initiated as part of legal proceedings and occur instead as routine clinical problems. ${ }^{2}$ Clinicians find capacity evaluations quite challenging to perform. ${ }^{3}$

It is this context that makes the analysis by Owen et $a l^{4}$ in this issue of the Journal particularly useful. They examined the interaction between the most widely cited criteria for capacity (understanding, appreciation and reasoning) with varying types of conditions impairing DMC, by comparing patients from a psychiatric hospital (55\% of whom had psychoses or mania) and a medical hospital (at least 25\% of whom had cognitive impairment with a Mini-Mental State Examination $<24)$. In the original studies from which the data were drawn, understanding,

†See pp. 461-467, this issue. appreciation and reasoning abilities were measured by an experienced psychiatrist using the MacArthur Competence Assessment Tool-Treatment (MacCAT-T), which yields dimensional scores for each ability; the MacCAT interview along with other available clinical information were then used by the psychiatrist to arrive at a categorical (yes $v$. no) determination of DMC. Although this retrospective analysis lacked some relevant clinical information (for example, cognition-related diagnoses among the medical in-patients), it still yielded important insights. I shall comment on three points, with an eye towards what the results might mean for future DMC research.

\section{Impaired understanding in evaluating DMC in both medical and psychiatric patients}

First, although it is tempting to emphasise the differences between the two patient groups' performance on the three criteria for $\mathrm{DMC}$, we should begin with what they have in common. The authors found that in both hospital groups, about a quarter had poor understanding (defined as $\leqslant 50 \%$ score on the understanding subscale); having poor understanding was a strong indicator of incapacity regardless of hospital group, with $81-93 \%$ lacking DMC. Those with poor understanding scores who lacked DMC generally also had poor appreciation and reasoning scores. This makes sense because if someone cannot absorb information then it is difficult to form beliefs about it or manipulate it (analogous to someone with impaired attention who appears to have poor short-term memory). Although it is not surprising that $67 \%$ of those with incapacity among medical patients had poor understanding (where one would expect cognitive dysfunction more than psychopathology to drive impairment), it is notable that almost half (44\%) of the psychiatric patients lacking decisional capacity had poor understanding. This is consistent with previous studies of psychiatric patients (those with chronic psychoses) showing that cognitive impairment, rather than classic positive psychotic symptoms, can be the primary source of incapacity for many people in this patient group. ${ }^{5,6}$ At any rate, clinicians should remain aware that lack of understanding accounts for nearly half of cases of incapacity even among psychiatric patients.

\section{What is the theoretical and empirical significance of impaired reasoning?}

Second, the theory behind DMC says that understanding is not sufficient for intact capacity and the authors confirmed this: 
$39 \%$ of psychiatric patients who showed good understanding nevertheless lacked DMC - virtually all of them had poor scores on appreciation, whereas only $17 \%$ had poor scores on reasoning. These are presumably patients who, despite their intellectual comprehension and intact reasoning, are unable to form adequate beliefs about their illness or treatment - a pattern attributable to their psychopathology (such as delusions) more than to cognitive impairment.

In contrast, among the medically ill, few patients with good understanding lacked DMC (13\%). Everyone (12 of 12) in this small group lacking DMC scored poorly on reasoning yet most (10 of 12) had good scores on the appreciation task. These patients who were apparently determined to be lacking in DMC solely on the basis of poor reasoning present somewhat of a puzzle because they do not fit neatly into our normative understanding of incapacity. A comprehensive legal review by the authors of the MacCAT instruments found that poor reasoning is not used by the courts as a sole basis for finding someone as incapable. ${ }^{7}$ There are several potential explanations for this apparent discrepancy.

It may be a reflection of the fact that operationalisation of the reasoning ability in the MacCAT instrument is based on psychological, rather than legal, concepts, such that an expectation of close tracking between historical legal findings and clinical determinations using MacCAT may not be warranted. ${ }^{2}$ Also, we do not know whether the clinicians themselves justified their determinations of these patients' status using the reasoning standard - perhaps, for instance, despite 'good' understanding scores, the clinicians focused on the patients' failure to understand or appreciate specific items of particular import or that a $50 \%$ threshold for a 'good' score is insufficiently sensitive to detect understanding impairment sufficient for incapacity (after all, the $50 \%$ cut-off is somewhat arbitrary and low). Or, finally, it could be that UK clinicians in fact do use reasoning as a standalone standard, unlike in US courts. An in-depth qualitative analysis of these patients' interviews may yield insights. It would be instructive to find out just what kind of reasoning deficiency is compatible with intact understanding and appreciation and yet serious enough to merit a determination of incapacity. In this way, an empirical analysis could inform our normative discussions.

\section{Research challenges arising from the context-specific nature of DMC}

Finally, this analysis provided an important opportunity to examine a key methodological question about 'real-world' DMC studies. This study had important strengths: the subjects were real patients and the DMC capacity assessed was for real treatment decisions (albeit not real time). Further, the authors had available well-validated categorical DMC judgments (which is surprisingly uncommon in DMC research), making it possible for them to assess the relationship between the dimensional aspect of individual abilities with a categorical outcome variable for DMC. The methodological question arises from the fact that DMC assessment must be tailored to a particular decision, taking into account key contextual factors. ${ }^{1,2}$ Thus, some argue that the level of abilities needed to consent to psychiatric hospital admission should be lower than that needed for other decisions. ${ }^{8}$ Also, whether patients consent to or refuse a treatment raises different risk-benefit considerations. ${ }^{1,2,9}$ Psychiatrists in fact use differential thresholds depending on the risk-benefit context of the decision. ${ }^{10}$ Thus, it is possible that a competent patient (agreeing to an in-patient admission) could actually have worse performance on ability measures than someone deemed incompetent (but refusing to undergo an operation that is minimally burdensome but lifesaving). This potential variability in the meaning of the main outcome variable could present a significant complication to 'real-world' DMC studies.

The results of the Owen et al analysis are reassuring in that decision-specific DMC judgments by clinicians, even for a variety of medical and psychiatric decisions, still allowed detection of important associations between levels of ability and categorical DMC status. It may be that the risk-benefit range of the decisions was not sufficiently wide enough to affect categorical judgments. Or perhaps patients with relatively intact capacity tended to agree with recommended treatment whereas the more impaired patients were more likely to refuse - in which case the associations between categorical status and dimensional performance scores would be accentuated. (This might also explain the remarkably high reliability of categorical judgments among the clinician evaluators in this study.) As we conduct more real-world studies using actual patients' categorical DMC status, this is an aspect of the modern theory of DMC that researchers should incorporate into their work by explicitly including, for example, the valence of the patient's choice (acceptance or refusal of recommended treatment) in the analysis.

\section{Conclusions}

The rigorous interplay between theoretical and empirical inquiry exhibited in the Owen et al paper provides important insights and serves as a model for a new generation of DMC research. The modern framework for DMC assessment is ethically appealing because of its function-based and decision-specific focus. But such a framework has, as it were, many complex moving parts that require careful theoretical and empirical study if we are to provide a valid and reliable knowledge-base for busy clinicians who are asked to conduct DMC evaluations.

\section{Acknowledgement}

The views expressed are the author's; they do not represent any position or policy of the National Institutes of Health, the Department of Health and Human Services or the US government.

Scott Y. H. Kim, MD, PhD, Department of Bioethics, 10 Center Drive, 1C118, Clinical Center, National Institutes of Health, Bethesda, Maryland 20892, USA. Email: scott.kim@nih.gov

First received 26 Apr 2013, final revision 6 Sep 2013, accepted 10 Oct 2013

\section{References}

1 Grisso T, Appelbaum PS. Assessing Competence to Consent to Treatment: A Guide for Physicians and Other Health Professionals. Oxford University Press, 1998.

2 Kim SYH. Evaluation of Capacity to Consent to Treatment and Research. Oxford University Press, 2010.

3 Seyfried L, Ryan KA, Kim SYH. Assessment of decision-making capacity: views and experiences of consultation psychiatrists. Psychosomatics 2013; 54: $115-23$.

4 Owen GS, Szmukler G, Richardson G, David AS, Raymont V, Freyenhagen F, et al. Decision-making capacity for treatment in psychiatric and medical in-patients: cross-sectional, comparative study. Br J Psychiatry 2013: 203: 461-7.

5 Palmer BW, Savla GN. The association of specific neuropsychological deficits with capacity to consent to research or treatment. J Int Neuropsychol Soc 2007; 13: 1047-59.

6 Carpenter Jr WT, Gold JM, Lahti AC, Queern CA, Conley RR, Bartko JJ, et al. Decisional capacity for informed consent in schizophrenia research. Arch Gen Psychiatry 2000; 57: 533-8. 
7 Berg JW, Appelbaum PS, Grisso T. Constructing competence: formulating standards of legal competence to make medical decisions. Rutgers Law Rev 1996; 48: 345-96.

8 Appelbaum BC, Appelbaum PS, Grisso T Competence to consent to voluntary psychiatric hospitalization: a test of a standard proposed by APA Psychiatr Serv 1998; 49: 1193-6.
9 Buchanan AE, Brock DW. Deciding for Others: The Ethics of Surrogate Decision Making. Cambridge University Press, 1989.

10 Kim SYH, Caine ED, Swan JG, Appelbaum PS. Do clinicians follow a risksensitive model of capacity determination? An experimental video survey. Psychosomatics 2006; 47: 325-9.

\title{
Karl Jaspers: 100 years of General Psychopathology
}

\author{
Femi Oyebode
}

I first read Jaspers' General Psychopathology as a trainee in Newcastle-upon-Tyne. I have returned to it as a resource for thinking through difficult concepts in psychopathology but mostly as a model of how to combine a humane yet exacting system of reasoning to psychiatry.

Karl Jaspers (1883-1969) was born into an upper-middle-class family. He was a sickly child but it was only in April 1901 that bronchiectasis was diagnosed. This was a lifelong condition that influenced his choice of career but also his outlook as he was not expected to live long. Jaspers studied medicine in Berlin, Göttingen and Heidelberg. At Heidelberg, he came into contact with many of the leading intellectuals of his day: Max Weber, Friedrich Gundolf, Georg Lukács, and Ernst Bloch. It was here, too, that he met Ernst Mayer whose sister, Gertrud, Jaspers married in 1910. Jaspers and Gertrud had remained in Heidelberg throughout the war years despite Gertrud being Jewish and it is a remarkable fact that they survived. Jaspers was retired as professor of philosophy at the end of September 1937 because of a Nazi law excluding from office any civil servant married to a Jew.

Jaspers is regarded as an existential philosopher influenced by Nietzsche and Kierkegaard. He is probably best known for The Question of German Guilt, a work dealing with the consequences of collaboration with the Nazis. He was a contemporary of Martin Heidegger, and influenced Hannah Arendt. Publication of General Psychopathology marked both the end of Jaspers' career in psychiatry and the beginning of his career in philosophy.

In the preface to the first edition, Jaspers wrote:

'. . . in psychopathology it is dangerous merely to learn the matter, our task is not to "learn psychopathology" but to learn to observe, ask questions, analyse and think in psychopathological terms. I would like to help the student to acquire a well-ordered body of knowledge, which will offer a point of departure for new observations and enable him to set freshly acquired knowledge in its proper place'.

It is clear from this quotation that Jaspers aimed for a methodology. His aim was to take an individual case and abstract from it broad concepts that assist in the description of distinguishable psychic phenomena such that these become communicable concepts. The special problem for psychopathology was that Man is not merely an animal but that he is conscious and self-aware.

Jaspers' influence in analysing the nature of explanatory theories and of causal explanations is less recognised. He exposed the risks to reasoning of analogies and metaphors in the construction of explanatory theories. He cites one fundamental error, namely that of transforming anatomical cerebral structures into fantastic mechanistic theories. This propensity remains with us even today.

It is apposite to ask whether psychopathology is still relevant. Whether Jaspers had said all that there was so say in 1913. General Psychopathology is often read as if it was a compendium of established (rigid) knowledge rather than a point of view that is open, flexible and provisional. And it is forgotten that Jaspers' real objective was to lay the foundations for an attitude, a method, rather than to foreclose inquiry.

Perhaps the need for psychopathology is even more urgent today, as the preoccupation with lists, check boxes and menus invariably leads towards a superficial and arid psychiatry where the subjective experience of the person who ought to be at the centre of our attention takes second place. At the same time, the most minimal dose of empathy, wonder or curiosity is deployed by the clinician.

This is the last of a series of articles to commemorate in this Journal the centenary of publication of Karl Jaspers' General Psychopathology. Other articles were published in January, February, April, June and August of this year. 